\title{
A SOLUTION OF HADAMARD'S PROBLEM FOR A RESTRICTED CLASS OF OPERATORS ${ }^{1}$
}

\author{
JOHN E. LAGNESE
}

The following note deals with the Hadamard problem, which is to determine all linear 2nd order differential operators for which Huygens' principle is valid in the sense of "Hadamard's minor premise" $([1$, p. 54.], see also $[2, \S 1])$. Examples of Huygens' operators are the ordinary wave operators

$$
\square_{n}=\sum_{i=1}^{n-1} \frac{\partial^{2}}{\partial x_{i}^{2}}-\frac{\partial^{2}}{\partial t^{2}}
$$

in an odd number $n-1 \geqq 3$ of space dimensions and those operators equivalent $\mathrm{t}^{2}$ to $\square_{n}$. These are the so-called trivial operators, and the famous "Hadamard conjecture" 3 claims that all Huygens' operators are trivial. Examples to the contrary show that this conjecture cannot be completely true (see [2]-[7]); it is, however, valid for real Huygens' operators with constant principal part in 4 independent variables. This result, which was first established by Matthisson [8], plays a decisive role in our present considerations.

In this note we will show that there is a sense in which Hadamard's conjecture is essentially correct for operators of the form

$$
L_{n}=\Delta_{n-1}-\frac{\partial^{2}}{\partial t^{2}}+c(t), \quad \Delta_{n-1}=\sum_{i=1}^{n-1} \frac{\partial^{2}}{\partial x_{i}^{2}}
$$

with analytic $c(t)$. The connection between such Huygens' operators and $\square_{n}$ is through the medium of certain nontrivial transformations of one Huygens' operator into another. These are the $l$-transforms first defined in [2] which, for convenience, we briefly recall here.

Presented to the Society, September 3, 1965 under the title Huygens' principle and Hadamard's conjecture; received by the editors February $28,1967$.

1 This research was supported by the National Science Foundation through Grant GP-4493.

2 Two operators are equivalent if each can be transformed to the other through (i) proper coordinate transformations, (ii) multiplication of the dependent variable by a nonzero scalar function and (iii) multiplication of the operator by a nonzero function. These three transformation groups, which preserve the Huygens' character of an operator, are called trivial transformations. No operator of the form (1) can be trivial unless $c(t) \equiv 0$ (see [5]).

3 This conjecture was in fact never asserted by Hadamard. (See Courant and Hilbert [9, p. 438].) 
Let $L_{n}$ be an operator of the form (1) with $c(t)$ analytic and $\not \equiv 0$ on an interval $I$, and let $\mu(t)$ be a nonzero solution of

$$
\ddot{\mu}+c(t) \mu=0 \quad(\cdot=d / d t)
$$

on an interval $I^{\prime} C I$. If we define the operators

$$
l_{\mu}=\partial / \partial t-\dot{\mu} / \mu, \quad l_{\mu}^{*}=-\partial / \partial t-\dot{\mu} / \mu,
$$

we may write for $t \in I^{\prime}, L_{n}=\Delta_{n-1}+l_{\mu}^{*} l_{\mu}$. Therefore $l_{\mu} L_{n}=\tilde{L}_{n} l_{\mu}$ where $\tilde{L}_{n}=\Delta_{n-1}+l_{\mu} l_{u}^{*}$ is called the $l_{\mu}$-transform (on $I^{\prime}$ ) of $L_{n}$. In [2], Theorem 1 , the following fundamental result was established.

THEOREM 1. Let $L_{n}$ be an analytic Huygens' operator of the form (1) and $\tilde{L}_{n}$ be the $l_{\mu}$-transform of $L_{n}$. Then $\tilde{L}_{n+2}$ is also a Huygens' operator.

Since for even $n \geqq 4, \square_{n}$ is a Huygens' operator, one may invoke this result to generate an infinite number of classes of nontrivial Huygens' operators.

The operator $L_{n}$ will be called l-equivalent to $\square_{n}$ if $L_{n}$ can be mapped to $\square_{n}$ by a finite number of $l$-transforms, that is, if for some nonnegative integer $q$ we have

$$
l_{\mu_{0}} l_{\mu_{1}} \cdots l_{\mu_{q}} L_{n}=\square_{n} l_{\mu_{0}} l_{\mu_{1}} \cdots l_{\mu_{q}}
$$

where

(4) $\ddot{\mu}+c(t) \mu_{q}=0, \quad\left(l_{\mu_{k}} l_{\mu_{k}}^{*}\right) \mu_{k-1}=0, \quad k=q, q-1, \cdots, 1$.

Our principal result may now be stated as

Theorem 2. Every Huygens' operator $L_{n}$ of the form (1) with analytic $c(t)$ is l-equivalent to $\square_{n}$.

A partial converse of Theorem 2 follows as a corollary of Theorem 1 , namely, if $L_{n}$ is $l$-equivalent to $\square_{n}$ through (3) and (4), then $L_{n}$ must be a Huygens' operator for even $n \geqq 2(q+3)$. In fact the relation $l_{\mu} L_{n}=\widetilde{L}_{n} l_{\mu}$ implies $l_{1 / \mu} \widetilde{L}_{n}=L_{n} l_{1 / \mu}$ where $1 / \mu$ satisfies $\left(l_{\mu} l_{\mu}^{*}\right) 1 / \mu=0$. Now set $L_{n}=L_{n}^{q+1}$ and denote by $L_{n}^{q-k}$ the $l_{\mu_{q-k}}$-transform of $L_{n}^{q-k+1}$, $k=0,1, \cdots, q$. Then $\square_{n}=L_{n}^{0}$ and $L_{n}^{q-\boldsymbol{k}+1}$ is the $l_{\lambda_{q-k}}$-transform of $L_{n}^{q-k}$ where $\lambda_{q-k}=1 / \mu_{q-k}$. We therefore have $l_{\lambda_{0}} \square_{n}=L^{1} l_{\lambda_{0}}$ and more generally

$$
l_{\lambda_{k}} \cdots l_{\lambda_{0}} \square_{n}=L_{n}{ }^{k+1} l_{\lambda_{0}} \cdots l_{\lambda_{k}}, \quad k=0, \cdots, q .
$$

Setting $k=q$ in (5), it then follows from Theorem 1 that $L_{n}$ must be a Huygens' operator for even $n \geqq 4+2(q+1)=2(q+3)$.

If $L_{n}$ is a Huygens' operator and $\tilde{L}_{n}$ its $l_{\mu}$-transform, it is not gen- 
erally true that $\tilde{L}_{n}$ is a Huygens' operator. However, one can establish the following result, upon which is based the proof of the theorem.

Lemma 1. Let $L_{n}, n \geq 6$, be a Huygens' operator of the form (1) with analytic $c(t)$. There exists $\mu(t) \neq 0$ satisfying (2) such that

(A) if $\widetilde{L}_{n}$ is the $l_{\mu}$-transform of $L_{n}$, then $\widetilde{L}_{n-2}$ is a Huygens' operator.

Moreover, $\mu(t)$ is uniquely determined by condition (A) up to a constant factor.

The proof of this lemma rests heavily on the Hadamard theory of elementary solution [1]. For the relevant facts of the theory we refer to $[2, \S 2]$. In particular we make use of the fact that if our operator $L_{n}$ is Huygens', the same is true for $L_{n+2 m}, m=1,2, \cdots$. Since the $l_{\mu}$-transforms do not depend on $n$, it follows that if Lemma 1 (or Theorem 2) holds for a certain value of $n$, it holds also for $n+2$, $n+4, \cdots$. We may therefore assume in our proofs, and we do so, that $n$ is such that $L_{n-2}$ is not a Huygens' operator.

Proof of Theorem 2. Assuming for the moment the validity of the lemma, let $L_{n}$ be a given Huygens' operator with analytic $c(t)$. If $n=4$, then we know from [8] that $L_{n}=\square_{n}$. For $n=6,8, \cdots$, set $q=(n-6) / 2$. According to the lemma, there is a function $\mu_{q}(t)$ satisfying (2) such that $L_{n}^{q}$, the $l_{\mu_{q}}$-transform of $L_{n}$, has the property that $L_{n-2}^{q}$ is a Huygens' operator. Explicitly we have

$$
L_{n}^{q}=\Delta_{n-1}+l_{\mu q} l_{\mu q}^{*} .
$$

If $n-2=4$, the proof is finished. Otherwise applying the lemma next to $L_{n}^{q}$, we obtain a function $\mu_{q-1}(t)$ satisfying $\left(l_{\mu_{q}} l_{\mu_{q}}^{*}\right) \mu_{q-1}=0$ such that the $l_{\mu_{q-1}}$-transform of $L_{n}^{q}$,

$$
L_{n}^{q-1}=\Delta_{n-1}+l_{\mu_{q-1}} l_{\mu_{q-1}}^{*}
$$

is still a Huygens' operator if $\Delta_{n-1}$ is replaced by $\Delta_{n-5}$. Composing these two $l$-transforms gives the identity

$$
l_{\mu_{q-1}} l_{\mu_{q}} L_{n}=l_{\mu_{q-1}} L_{n}^{q} l_{\mu_{q}}=L_{n}^{q-1} l_{\mu_{q-1}} l_{\mu_{q}} .
$$

Continuing in this fashion, we obtain a sequence of operators $l_{\mu_{k}}$, $k=q, q-1, \cdots, 0$, defined by

$$
\ddot{\mu}_{q}+c(t) \mu_{q}=0, \quad\left(l_{\mu_{k}} l_{\mu_{k}}^{*}\right) \mu_{k-1}=0, \quad k=q, q-1, \cdots, 1,
$$

such that

$$
l_{\mu_{0}} l_{\mu_{1}} \cdots l_{\mu_{q}} L_{n}=L_{n}^{0} l_{\mu_{0}} l_{\mu_{1}} \cdots l_{\mu_{q}}
$$


and such that $L_{4}^{0}$ is a Huygens' operator. Again from [8] we conclude that $L_{4}^{0}=\square_{4}$ and thus $L_{n}^{0}=\square_{n}$, proving the theorem.

Proof of Lemma 1. Let $L_{n}, n \geqq 6$ be a fixed Huygens' operator with $c(t)$ analytic on the interval $I$. In this case, the elementary solution (in the sense of Hadamard) of $L_{n} u=0$ relative to a point $\tau \in I$, $\xi \in R^{n-1}$, is of the form

$$
\Gamma^{-(p+1)} \sum_{\nu=0}^{p} \beta_{\nu}(n) U_{\nu}(t, \tau) \Gamma^{\nu}+R, \quad p=\frac{n-4}{2},
$$

where $\Gamma=\sum_{i=1}^{n=1}\left(x_{i}-\xi_{i}\right)^{2}-(t-\tau)^{2}, R$ is some regular function and

$$
\begin{aligned}
& \beta_{0}=1, \quad \beta_{\nu}(n)=\alpha_{1} \alpha_{2} \cdots \alpha_{\nu}, \\
& \alpha_{\nu}=1 / 4(p-\nu+1), \quad \nu \neq p+1, \quad \alpha_{p+1}=\frac{1}{4} .
\end{aligned}
$$

The regular functions $U_{\nu}(t, \tau)$ are uniquely determined throughout $I$ and depend only on the form of $c(t)$ and not on $n$. In particular, $U_{p}(t, \tau)$ is a solution of $L_{n} u=0$ and hence satisfies (2) identically in $(t, \tau) \in I \times I$. We assume $U_{p}(t, \tau) \not \equiv 0$, which is equivalent to the assumption that $L_{n-2}$ is not a Huygens' operator.

The construction of the solution $\mu(t)$ is via the coefficient $U_{p}(t, \tau)$. In fact, since $U_{p}$ satisfies (2) the $l_{U_{p}}$-transform, say $\tilde{L}_{n}$, of $L_{n}$ is defined. Part (A) of Lemma 1 is established by proving first of all that $U_{p}(t, \tau)$ has the form ${ }^{4} U_{p}(t, \tau)=$ (const) $a_{p}(t) a_{p}(\tau)$ (so that $\tilde{L}_{n}$ does not depend on $\tau$ ). We then verify that $\widetilde{L}_{n-2}$ defined in this way (or equivalently, via the solution $\left.a_{p}(t)\right)$ is indeed a Huygens' operator. The verification of the formula above for $U_{p}(t, \tau)$ constitutes the main part of the proof.

As the $U_{\nu}(t, \tau)$ are independent of $n$, they may be used to form the elementary solution of $L_{m} u=0$ for any integer $m \geqq 2$. In particular, for $m=2$ the elementary solution is $W \log \bar{\Gamma}+\bar{R}$ where

$$
\begin{aligned}
W(t, \tau, r) & =\sum_{\nu=0}^{p} \beta_{\nu} U_{\nu}(t, \tau)\left(r^{2}-T^{2}\right)^{\nu}, \\
\beta_{\nu} & =\beta_{\nu}(2), \quad \bar{\Gamma}=r^{2}-T^{2}=\left(x_{1}-\xi_{1}\right)^{2}-(t-\tau)^{2} .
\end{aligned}
$$

Hadamard has shown [1, pp. 71, 72] that $W$ is identical with the regular Riemann function of $L_{2}$. Thus $W$ is a regular solution of $L_{2} u=0$.

${ }^{4}$ The fact that $L_{n}$ is formally selfadjoint implies the symmetry relation $U_{\nu}(t, \tau)$ $=U_{\nu}(\tau, t)$. Since moreover $U_{p}$ satisfies (2), it must have the form $U_{p}(t, \tau)=k_{11} \mu(t) \mu(\tau)$ $+k_{12}(\mu(t) \lambda(\tau)+\mu(\tau) \lambda(t))+k_{22} \lambda(t) \lambda(\tau)$ where $(\mu, \lambda)$ is any linearly independent pair of solutions of $(2)$ and $\left(k_{i j}\right)$ is a symmetric $2 \times 2$ matrix of constants. The statement $U_{p}(t, \tau)=k a_{p}(t) a_{p}(\tau)$ is equivalent to rank $\left(k_{i j}\right)=1$. 
We now define for real values of $\rho$ the function

$$
\hat{u}(t, \tau, \sigma)=\frac{\eta(T)}{2} \int_{-T}^{T} W(t, \tau, r) e^{-2 \pi i \rho r} d r, \quad \sigma=\frac{1}{2 \pi i \rho}
$$

where $\eta(T)$ is equal to 1 or zero depending on whether $T \geqq 0$ or $<0$. Then $\hat{u}$ is the fundamental solution for the ordinary differential operator

$$
\hat{L}=d^{2} / d t^{2}+\left(c(t)-1 / \sigma^{2}\right)
$$

that is, $\hat{u}$ satisfies

(a) $d a / d t$ and $d^{2} a / d t^{2}$ are continuous with respect to $t$ in $I$ except at $t=\tau$, where $\left(d^{+} / d t-d^{-} / d t\right) \hat{u}=1$,

(b) $\hat{L} a=0$ in $I$ except at $t=\tau$,

(c) $a \equiv 0$ for $t<\tau$.

The conditions (a), (b) and (c) imply the uniqueness of the fundamental solution. Replacing $W$ in (7) through (6) gives

$$
u(t, \tau, \sigma)=\frac{1}{2} \eta(T) \sum_{\nu=0}^{p} \beta_{\nu} U_{\nu}(t, \tau) \int_{-T}^{T}\left(r^{2}-T^{2}\right)^{\nu} e^{-2 \pi i \rho r} d r .
$$

The integral can be expressed as

$$
\begin{aligned}
\int_{-T}^{T}\left(r^{2}-T^{2}\right)^{\nu} e^{-2 \pi i \rho r} d r & =(-1)^{\nu} T^{\nu+1 / 2} \frac{\nu ! \pi^{1 / 2}}{(\pi \rho)^{\nu+1 / 2}} J_{\nu+1 / 2}(2 \pi \rho T) \\
& =2 \sigma \operatorname{Im}\left(e^{T / \sigma} \sum_{k=0}^{\nu} A_{k}^{\nu} T_{\sigma^{\nu-k}}^{\nu+k}\right)
\end{aligned}
$$

where $\operatorname{Im}(z)=$ imaginary part of $z, J_{v+1 / 2}$ is the Bessel function of first kind of order $\nu+1 / 2$ and

Thus

$$
A_{k}^{\nu}=\frac{(-1)^{\nu+k} \nu !(\nu+k) !}{(\nu-k) !} 2^{\nu-k}, \quad k=0,1, \cdots, \nu .
$$

$$
\begin{aligned}
\mathfrak{u}(t, \tau, \sigma) & =\sigma \eta(T) \sum_{\nu=0}^{p} \beta_{\nu} U_{\nu}(t, \tau) \operatorname{Im}\left(e^{T / \sigma} \sum_{k=0}^{\nu} A_{k}^{\nu} T_{\sigma^{\nu-k}}^{\nu+k}\right) \\
& =\sigma \eta(T) \operatorname{Im}\left(e^{T / \sigma} \sum_{\nu=0}^{2 p} \hat{\bigcup}_{\nu}(t, \tau) \sigma^{\nu}\right)
\end{aligned}
$$

with

$$
\begin{aligned}
\hat{O}_{2 \nu}(t, \tau) & =\sum_{k=0}^{\nu} \beta_{\nu+k} A_{\nu-k}^{\nu+k} U_{\nu+k}(t, \tau) T^{2 k}, \quad \nu=0, \cdots, p, \\
\hat{O}_{2 \nu+1}(t, \tau) & =T \sum_{k=0}^{\nu} \beta_{\nu+k+1} A_{\nu-k}^{\nu+k+1} U_{\nu+k+1}(t, \tau) T^{2 k}, \quad \nu=0, \cdots, p-1 .
\end{aligned}
$$


In particular, since $U_{\nu} \equiv 0$ for $\nu>p$,

$$
\hat{U}_{2 p}(t, \tau)=\beta_{p} A_{p}^{p} U_{p}(t, \tau) \not \equiv 0 .
$$

We next show that $\hat{O}_{2 p}(t, \tau)=a_{p}(t) a_{p}(\tau)$ where $a_{p}(t)$ is some solution of (2). In view of (8), $\hat{L} u=0$ admits a solution in $I$ of the form $u(t, \sigma)=e^{t / \sigma} \sum_{v=0}^{r} b_{\nu}(t) \sigma^{\nu}$ where the $b_{\nu}$ are real and $b_{r}(t) \not \equiv 0$. We shall call the integer $r$ the degree of $u(t, \sigma)$. Let

$$
u_{m}(t, \sigma)=e^{t / \sigma} \sum_{\nu=0}^{q} a_{\nu}(t) \sigma^{\nu} \equiv e^{t / \sigma} P_{m}(t, \sigma)
$$

be the minimal solution of $\hat{L} u=0$, that is, the solution of least degree. The coefficients $a_{v}(t)$ of $P_{m}$ satisfy

$$
\dot{a}_{0}=0, \quad \dot{a}_{\nu}=-\frac{1}{2}\left(\ddot{a}_{v-1}+c(t) a_{\nu-1}\right), \quad \nu \geqq 1 .
$$

It is easy to verify that every solution in $I$ of the form $e^{t / \sigma} \sum_{\nu=0}^{r} b_{\nu}(t) \sigma^{\nu}$ is a multiple of $u_{m}$ by a polynomial in $\sigma$ with constant coefficients. With the normalization $a_{0} \equiv 1$, the minimal solution is clearly unique. We note that the coefficient $a_{q}(t)$ in $u_{m}$ satisfies $\ddot{a}_{q}+c(t) a_{q}=0$.

The fundamental solution $\hat{u}$ of $\hat{L} u=0$ may be expressed in terms of $u_{m}$ as

$$
\begin{aligned}
u(t, \tau, \sigma) & =\eta(T) \frac{u_{m}(t, \sigma) \bar{u}_{m}(\tau, \sigma)-\bar{u}_{m}(t, \sigma) u_{m}(\tau, \sigma)}{\mathscr{W}\left(u_{m}, \bar{u}_{m}\right)} \\
& =2 n(T) \operatorname{Im}\left[e^{T / \sigma} P_{m}(t, \sigma) \bar{P}_{m}(\tau, \sigma)\right] / W\left(u_{m}, \bar{u}_{m}\right)
\end{aligned}
$$

where $\bar{u}_{m}=$ complex conjugate of $u_{m}$ and the Wronskian

$$
\begin{aligned}
W\left(u_{m}, \bar{u}_{m}\right) & =\bar{u}_{m}(t, \sigma) \frac{d u_{m}}{d t}(t, \sigma)-u_{m}(t, \sigma) \frac{d \bar{u}_{m}}{d t}(t, \sigma) \\
& =\frac{2}{\sigma}\left[P_{m} \bar{P}_{m}+\frac{\sigma}{2}\left(\bar{P}_{m} \frac{d P_{m}}{d t}-P_{m} \frac{d \bar{P}_{m}}{d t}\right)\right]
\end{aligned}
$$

is independent of $t$. The expression in brackets in (11) is both real and a polynomial in $\sigma$, and therefore

$$
W\left(u_{m}, \bar{u}_{m}\right)=\frac{2}{\sigma} \sum_{v=0}^{q} k_{2 v} \sigma^{2 v}
$$

with $k_{0}=1, k_{2 \nu}=$ const, $\nu=1, \cdots, q$. On account of the uniqueness of $\mathfrak{u}$ it follows from (8), (10) and (12) that

$$
\sum_{\nu=0}^{2 p} \hat{O}_{\nu}(t, \tau) \sigma^{\nu}=P_{m}(t, \sigma) \bar{P}_{m}(\tau, \sigma) / \sum_{\nu=0}^{q} k_{2 \nu} \sigma^{2 \nu}
$$


Since $u_{m}$ is the minimal solution of $\hat{L} u=0$, neither $P_{m}$ nor $\bar{P}_{m}$ can admit a polynomial divisor with constant coefficients and so we must have

$$
\begin{aligned}
& q=p, \quad k_{2 v}=0, \quad \nu=1,2, \cdots, p, \\
& \hat{O}_{2 p}(t, \tau)=a_{p}(t) a_{p}(\tau)=\beta_{p} A_{p}^{p} U_{p}(t, \tau) \not \equiv 0 .
\end{aligned}
$$

The formula (13) establishes the representation of the coefficient $U_{p}$ which was mentioned at the beginning of the proof. Moreover, we have proved the following:

Lemma 2. Let $L_{n}=\square_{n}+c(t)$ be a Huygens' operator with analytic $c(t)$. Then the equation $\hat{L} u=\ddot{u}+\left(c(t)-1 / \sigma^{2}\right) u=0$ admits a solution of the form

$$
u(t, \sigma)=e^{t / \sigma} \sum_{\nu=0}^{p} a_{\nu}(t) \sigma^{\nu}, \quad p=\frac{n-4}{2}
$$

If, moreover, $L_{n-2}$ is not a Huygens' operator, then $u(t, \sigma)$ is the minimal solution of $\hat{L} u=0$.

To complete the proof of (A) of Lemma 1 , set $\mu(t)=a_{p}(t)$ and let $\tilde{L}_{n}$ be the $l_{\mu}$-transform of $L_{n}$. We will show that $\tilde{L}_{n-2}$ is a Huygens' operator.

Let $m$ denote the smallest number of independent variables in which $\widetilde{L}_{m}$ is a Huygens' operator. As $L_{n-2}$ is not a Huygens' operator but is the $l_{1 / u}$-transform of $\tilde{L}_{n-2}$, from Theorem 1 follows that $m$ $\geqq n-2$. To prove $m=n-2$ it suffices, by Lemma 2 , to show that $\check{L} u=-\left(l_{\mu} l_{\mu}^{*}+1 / \sigma^{2}\right) u=0$ admits a solution of degree $p-1$.

But from $l_{\mu} L_{n}=\tilde{L}_{n} l_{\mu}$ we evidently have

$$
l_{\mu} \hat{L}=\check{L} l_{\mu}
$$

and therefore $\check{L}\left(l_{\mu} u_{m}(t)\right)=0$. Explicitly

$$
l_{\mu} u_{m}(t)=\frac{1}{\sigma} e^{t / \sigma} \sum_{\nu=0}^{p+1}\left(a_{\nu}+\dot{a}_{\nu-1}-a_{\nu-1} \frac{\dot{a}_{p}}{a_{p}}\right) \sigma^{\nu} .
$$

The coefficient of $\sigma^{p+1}$ in (14) is $\dot{a}_{p}-a_{p}\left(\dot{a}_{p} / a_{p}\right) \equiv 0$. We have to show that the coefficient of $\sigma^{p}$ also vanishes identically, i.e.

$$
a_{p}+\dot{a}_{p-1}-a_{p-1}\left(\dot{a}_{p} / a_{p}\right) \equiv 0 .
$$

But (15) is a special case of a general recursion formula satisfied by the coefficients $a_{\nu}$ in $u_{m}$. In fact, by substituting the right-hand side of 
(9) into the right member of (11) and then using (12) and (13) we obtain

$$
2 a_{2 \nu}=2 \sum_{k=1}^{\nu-1}(-1)^{k+1} a_{k} a_{2 \nu-k}+\sum_{k=0}^{2 \nu-1}(-1)^{k+1} a_{k} \dot{a}_{2 \nu-k-1}+(-1)^{\nu+1} a_{\nu}^{2}
$$

valid for $\nu=1,2, \cdots$, where $a_{\nu} \equiv 0$ for $\nu>p$. Setting $\nu=p$ in (16) gives immediately the identity (15). Thus $\sigma l_{\mu} u_{m}$ is a solution of $\check{L} u=0$ having degree $p-1$.

To prove the uniqueness part of Lemma 1 , let $\mu(t)$ and $\lambda(t)$ be two nontrivial solutions of (2) for which condition (A) is satisfied, that is, if $\tilde{L}_{n}$ and $\bar{L}_{n}$ are the $l_{\mu}$ - and $l_{\lambda}$-transforms of $L_{n}$, respectively, then both $\tilde{L}_{n-2}$ and $\bar{L}_{n-2}$ are Huygens' operators. Since $L_{n-2}$ is the $l_{1 / \mu}$-transform of $\widetilde{L}_{n-2}$, the coefficient $U_{p}(t, \tau)$ in the elementary solution of $L_{n} u=0$ is given in terms of $\mu$ by $U_{p}(t, \tau)=k_{\mu}(t) \mu(\tau), k=$ const., as was shown in $[2, \S 3]$. Also, $L_{n-2}$ is the $l_{1 / \lambda}$-transform of $\bar{L}_{n-2}$ so that $U_{p}(t, \tau)$ $=\bar{k} \lambda(t) \lambda(\tau)$. It follows from the uniqueness of $U_{p}$ that $k \mu(t) \mu(\tau)$ $=\bar{k} \lambda(t) \lambda(\tau)$, and since $U_{p} \not \equiv 0$ neither $\tilde{k}$ nor $\bar{k}$ can vanish. Therefore $\mu(t)=($ const $) \lambda(t)$ and the proof of Lemma 1 is complete.

Acknowledgment. The author would like to thank Professor K. L. Stellmacher for several stimulating discussions and a number of helpful suggestions.

\section{REFERENCES}

1. J. Hadamard, Lectures on Cauchy's problem in linear partial differential equations, Yale Univ. Press, New Haven, Conn., 1923.

2. J. E. Lagnese and K. L. Stellmacher, A method of generating classes of Huygens' operators, J. Math. Mech. 17 (1967), 461-472.

3. J. E. Lagnese, A new differential operator of the pure wave type, J. Differential Equations 1 (1965), 171-187.

4. K. Stellmacher, Ein Beispiel einer Huyghensschen Differentialgleichung, Nachr. Akad. Wiss. Göttingen Math.-Phys. Kl. 10 (1953), 133-138.

5. - Eine Klasse Huyghensscher Differentialgleichungen und ihre Integration, Math. Ann. 130 (1955), 219-233.

6. - Das Huyghenssche Prinzip fïr hyperbolische Differentialgleichungen mit konstant Haupteil, Math. Z. (to appear).

7. P. Günther, Ein Beispiel einer nichttrivialen Huyghensschen Differentialgleichungen mit vier unabhängigen Variablen, Arch. Rational Mech. Anal. 18 (1965), 103-106.

8. M. Matthisson, Le problème de M. Hadamard relatif à la diffusion des ondes, Acta Math. 71 (1939), 249-282.

9. R. Courant and D. Hilbert, Methoden der mathematischen Physik. II, SpringerVerlag, Berlin, 1937.

GEORGETOWN UNIVERSITY 